\title{
ALMOST PERIODIC SOLUTIONS TO SYSTEMS OF PARABOLIC EQUATIONS
}

\author{
JANPOU NEE \\ Academia Sinica \\ Institute of Mathematics \\ Nankang, Taipei, 11529, Taiwan, ROC
}

(Received February, 1994; revised May, 1994)

\begin{abstract}
In this paper we show that the second-order differential solution is $\mathbb{L}^{2}$-almost periodic, provided it is $\mathbb{L}^{2}$-bounded, and the growth of the components of a nonlinear function of a system of parabolic equation is bounded by any pair of consecutive eigenvalues of the associated Dirichlet boundary value problems.
\end{abstract}

Key words: Almost Periodic Solutions, System of Nonlinear Parabolic Equations.

AMS (MOS) subject classifications: $35 \mathrm{~B} 15,35 \mathrm{~K} 55,35 \mathrm{~K} 99$.

\section{Introduction}

Foais et al. [2] proved that if a solution of some system of parabolic equations in $C^{2}(\bar{\Omega})$ and $L^{2}$-bounded satisfying certain conditions then it is a $L^{2}$-almost periodic solution.

Recall that a continuous function $f: \mathbb{R} \rightarrow X$ is $X$-almost periodic if for every $\epsilon$ there is a relatively dense subset $T_{\epsilon} \subset \mathbb{R}$ such that

$$
\sup _{t}\|f(t+\tau)-f(t)\|_{X}<\epsilon, \quad \forall \tau \in T_{\epsilon},
$$

where $X$ is some Banach space.

Recently, Corduneanu [1] and Yang [4] extended the results of Foias to nonlinear parabolic equations. In this paper we extend the results of Corduneanu [1] and Yang [4] to the following system of nonlinear parabolic equations,

$$
\left\{\begin{array}{c}
\partial_{t} u=\Delta u+f(t, x, u) \\
\left.u\right|_{\partial \Omega}=0 \\
u(0, x)=u_{0}
\end{array}\right.
$$

where $u$, and $f \in \mathbb{R}^{m}$ are $m$-vector valued functions, $\partial_{t}=\frac{\partial}{\partial}$, and $\Omega$ is some bounded domain in $\mathbb{R}^{n}$ with sufficiently smooth boundary $\partial \Omega$. Moreover, we assume that $f: \mathbb{R} \times \Omega \times \mathbb{R}^{m} \rightarrow \mathbb{R}^{m}$ satisfies the following conditions (cf. $[1,4])$ : 
(CI) $f(t, x, u)$ is continuous and $\mathbb{L}^{2}$-almost periodic in $t$, and uniformly continuous with respect to $u_{j}$.

(CII) The matrix $\mathbf{D}(f)=\left(f_{i, j}\right)$ is diagonalizable, with eigenvalues $\mu_{j}$, and for every $j=1,2, \ldots, m$, there exists some integer $i(j)$ such that $\lambda_{i(j)-1}<\mu_{j}<\lambda_{i(j)}$.

Here $f_{i, j}=\frac{\partial f_{i}}{\partial u_{j}}$, and $\mathbb{L}^{2}=L^{2}(\Omega) \times \cdots \times L^{2}(\Omega), m$-times. We call matrix $\mathbf{D}(f)$ diagonalizable if there exists a nonsingular matrix $M$ such that $M \mathrm{D}(f) M^{-1}=I$, at every triple $(t, x, u)$, where $I$ is the identity matrix. Similarly, $M$ is nonsingular if $\operatorname{det} M \neq 0$ and $\mu$ is an eigenvalue of matrix $\mathbf{D}(f)$ if $\operatorname{det}(\mathbf{D}(f)-\mu)=0$. Notice that condition $(C I I)$ implies that $\mu_{j}>0$, since $\lambda_{j}$ is the eigenvalues of Laplacian in the domain $\Omega$ corresponding to the eigenfunction $\phi_{j}$, which satisfies

$$
\left\{\begin{array}{c}
\Delta \phi_{j}+\lambda_{j} \phi_{j}=0 \\
\left.\phi_{j}\right|_{\partial \Omega}=0
\end{array}\right.
$$

We arrange $\lambda_{j}$ in the ascending order

$$
0<\lambda_{1}<\lambda_{2} \leq \lambda_{3} \ldots \text { for } j=1,2, \ldots
$$

To simplify the notation, we use $\lambda_{0}$ to denote 0 , and the function space $\mathbb{C}^{2}(\bar{\Omega})=$ $C^{2}(\bar{\Omega}) \times \cdots \times C^{2}(\bar{\Omega}), m$-times.

\section{Main Result}

Before we prove the main theorem of this paper we first derive a useful a priori estimate of the following problem,

$$
\left\{\begin{array}{c}
\partial_{t} w-(\Delta+D) w=v \\
\left.u\right|_{\partial \Omega}=0
\end{array}\right.
$$

where $w, v$ are $m$-vector valued functions, $D=\left(\delta_{i, j} \nu_{j}\right)$ is a diagonal matrix, and $\delta_{i, j}$ is the Kronecker delta, $\nu_{j}$ are positive real numbers satisfying $\lambda_{i(j)-1}<\nu_{j}<\lambda_{i(j)}$. Here $i(j)$ is the same as in the condition $(C I I)$.

Lemma 1: Let $w, v \in \mathbb{C}^{2}(\bar{\Omega})$ be $\mathbb{L}^{2}$-bounded satisfying problem (3). If $\nu_{j}$ satisfies the assumption above, then

$$
\sup _{t} \int_{\Omega} w_{j}^{2}(t, x) d x \leq \max \left\{\left(\nu_{j}-\lambda_{i(j)-1}\right)^{-2},\left(\nu_{j}-\lambda_{i(j)}\right)^{-2}\right\} \sup _{t} \int_{\Omega} v_{j}^{2}(t, x) d x .
$$

Proof: It is well known that $\left\{\phi_{j}\right\}_{j=1}^{\infty}$ form an orthogonal basis of $L^{2}(\Omega)$, thus we have

$$
\begin{aligned}
w_{j}(t, x) & =\sum_{k} a_{j, k} \phi_{k}, \\
v_{j}(t, x) & =\sum_{k} b_{j, k} \phi_{k} .
\end{aligned} \quad \text { for } j=1,2, \ldots, m
$$

The Parseval formula and the assumption of $L^{2}(\Omega)$-boundedness imply that 


$$
\begin{aligned}
& \sum_{j, k} a_{j, k}^{2}=\int_{\Omega} w^{2} \leq c \\
& \sum_{j, k} b_{j, k}^{2}=\int_{\Omega} v^{2} \leq c
\end{aligned}
$$

for some positive constant $c$ which is independent of $t$.

Substituting equation (5) into equation (3) yields

$$
a_{j, k}^{\prime}(t)+\left(\lambda_{k}-\nu_{j}\right) a_{j, k}(t)=b_{j, k}(t),
$$

for $j=1, \ldots, m, k=1,2, \ldots$. Thus for any $t_{0} \in \mathbb{R}$ we have

$$
a_{j, k}(t)=e^{-\left(\nu_{j}-\lambda_{k}\right)\left(t_{0}-t\right)} a_{j, k}\left(t_{0}\right)+\int_{t_{0}}^{t} e^{-\left(\nu_{j}-\lambda_{k}\right)(s-t)} b_{j, k}(s) d s .
$$

Since $\lambda_{i(j)-1}<\nu_{j}<\lambda_{i(j)}$, we have $\nu_{j}-\lambda_{k}>0$ for $k \leq i(j)-1$. Thus for $t_{0}>t$, the following is true

$$
\left|a_{j, k}(t)\right| \leq e^{-\left(\nu_{j}-\lambda_{k}\right)\left(t_{0}-t\right)}\left|a_{j, k}\left(t_{0}\right)\right|+\frac{1-e^{-\left(\nu_{j}-\lambda_{k}\right)\left(t_{0}-t\right)}}{\nu_{j}-\lambda_{k}}\left|b_{j, k}(t)\right| .
$$

Using (5), (6), and the fact that $a_{j, k}, b_{j, k}$ are bounded functions of $t$, and letting $t_{0} \rightarrow \infty$, the above inequality yields

$$
\sup _{t}\left|a_{j, k}(t)\right| \leq \frac{1}{\nu_{j}-\lambda_{k}} \sup _{t}\left|b_{j, k}(t)\right| .
$$

Similarly, the above inequality is true for $k \geq i(j)$ which implies

$$
\sup _{t}\left|a_{j, k}(t)\right| \leq \alpha_{j} \sup _{t}\left|b_{j, k}(t)\right|
$$

where

$$
\alpha_{j}=\max \left\{\frac{1}{\nu_{j}-\lambda_{i(j)-1}}, \frac{1}{\lambda_{i(j)}-\nu_{j}}\right\}
$$

Thus the assertion of the lemma holds.

Theorem 2: If $u$ is a $\mathbb{C}^{2}(\bar{\Omega}), \mathbb{L}^{2}$-bounded solution of problem (1), and if $f_{j, i}$ is a continuous function satisfying conditions $(C I)$ and $(C I I)$, then $u$ is $\mathbb{L}^{2}$-almost periodic.

Proof: Let $u$ be a solution of equation (1), then for a given $\tau \in \mathbb{R}$ we define the vector valued function $w=u(t+\tau, x)-u(t, x)$. Then $w$ satisfies the following equation,

$$
\left\{\begin{array}{c}
w_{t}-\Delta w=f(t+\tau, w, x, u(t+\tau, x))-f(t, x, u(t, x)) \\
\left.w\right|_{\partial \Omega}=0
\end{array}\right.
$$

Applying the mean value theorem to $f_{j}$ with respect to the component $u_{i}$ and letting $\alpha_{j, i}$ be a constant in the interval $(0,1)$, we have that

$$
\psi_{j, i}=\alpha_{j, i} u_{i}(t+\tau, x)+\left(1-\alpha_{j, i}\right) u_{i}(t, x)
$$


satisfies

$$
\begin{gathered}
f_{j}\left(t, x, u_{1}(t, x), \ldots, u_{i-1}(t, x), u_{i}(t+\tau, x), \ldots, u_{m}(t+\tau, x)\right) \\
-f_{j}\left(t, x, u_{1}(t, x), \ldots, u_{i}(t, x), u_{i+1}(t+\tau, x), \ldots, u_{m}(t+\tau, x)\right) \\
=f_{j, i}\left(t, x, u_{1}(t, x), \ldots, \psi_{i}, u_{i+1}(t+\tau, x), \ldots, u_{m}(t+\tau, x)\right) w_{i}(t, x) .
\end{gathered}
$$

Let the vector valued functions $\Psi_{j, i}$ be

$$
\Psi_{j, i}=\left(u_{1}(t, x), \ldots, u_{i-1}(t, x), \psi_{j, i}, u_{i+1}(t+\tau, x), \ldots, u_{m}(t+\tau, x)\right),
$$

then $w_{j}$ satisfies

$$
\begin{aligned}
& \partial_{t} w_{j}-\Delta w_{j}=\sum_{i} f_{j, i}\left(t+\tau, x, \Psi_{i}\right) w_{i} \\
& +f_{j}(t+\tau, x, u(t, x))-f_{j}(t, x, u(t, x)),
\end{aligned}
$$

and boundary condition

$$
\left.w_{j}\right|_{\partial \Omega}=0
$$

Since $w_{j}$ are $L^{2}(\Omega)$-bounded, we have

$$
w_{j}(t, x)=\sum_{k} a_{j, k}(t) \phi_{k}
$$

for $j=1, \ldots, m$. The condition $(C I I)$ implies that for every $j$ there exist two constants $\bar{\theta}_{j}$ and $\widehat{\theta}_{j}$, and some integer $i(j) \geq 1$ such that

$$
\lambda_{i(j)-1}<\bar{\theta}_{j} \leq \mu_{j} \leq \widehat{\theta}_{j}<\lambda_{i(j)}
$$

Recall that $\lambda_{0}$ is 0 .

Equation (9) can be rewritten as

$$
\begin{gathered}
\partial_{t} w_{j}-\left(\Delta w_{j}+\nu_{j}\right)=\sum_{\substack{i=1 \\
i \neq j}} f_{j, i}\left(t+\tau, x, \psi_{i}\right) w_{i}+\left(f_{j, j}-\nu_{j}\right) w_{j} \\
+f_{j}(t+\tau, x, u(t, x))-f_{j}(t, x, u(t, x))
\end{gathered}
$$

where $\bar{\theta}_{j}<\nu_{j}<\widehat{\theta}_{j}$. Observe that the $\nu_{j}$ are real; and they will be determined later. By inequality (8), we immediately obtain

where

$$
\sup _{t}\left(\left(1-k_{j}\right)\left|w_{j}\right|-\sum_{\substack{i \neq 1 \\ i \neq j}} \beta_{j, i}\left|w_{i}\right|\right) \leq \gamma_{j} s_{t} p\left|v_{j}\right|
$$

$$
k_{j}=\frac{\left|f_{j, j}-\nu_{j}\right|}{\alpha_{j}}, \beta_{j, i}=\frac{\left|f_{j, i}\right|}{\alpha_{j}}, \gamma_{j}=\frac{1}{\alpha_{j}}, \alpha_{j}=\max \left\{\frac{1}{\nu_{j}-\lambda_{i(j)}-1}, \frac{1}{\lambda_{i(j)}-\nu_{j}}\right\}
$$

for $j=1, \ldots, m$. Let $\epsilon_{i}>0$, satisfy

$$
\sup _{t}\left\{\left(1-k_{j}\right)\left|w_{j}\right|=\sum_{\substack{i \neq 1 \\ i \neq j}} \beta_{j, i}\left|w_{i}\right|\right\}=\gamma_{j} \sup _{t}\left|v_{j}\right|+\epsilon_{i} .
$$


Let $\xi=\left(\epsilon_{1}, \ldots, \epsilon_{m}\right)$, and rewrite the above equation as

where

$$
M \cdot w=\xi+G \cdot v
$$

$$
w=\left(\sup _{t}\left|w_{1}\right|, \ldots, \sup _{t}\left|w_{m}\right|\right), \quad v=\left(\sup _{t}\left|v_{1}\right|, \ldots, \sup _{t}\left|v_{m}\right|\right),
$$

$M=\left(m_{i, j}\right), G=\left(\delta_{i, j} \gamma_{j}\right)$ are $m \times m$ matrices, where $m_{i, j}=\beta_{i, j}$, for $j \neq i$, and $m_{j, j}=1-k_{j}$. Since $\bar{\theta}_{j}<\nu_{j}<\hat{\theta}_{j}$, and using condition $(C I I)$, we may choose suitable $\nu_{j}$ such that $1-k_{j}>0$, and $M$ is diagonalizable. By linear algebra we have

$$
w=\xi+G \cdot w
$$

Since $\xi=\left(\epsilon_{1}, \ldots, \epsilon_{m}\right)$ and $\epsilon_{i}>0$, we have

$$
\sup _{t} \int_{\Omega} w_{j}^{2}(t, x) d x \leq c_{j} \sup _{t} \int_{\Omega} v_{j}^{2}(t, x) d x, \text { for } j=1, \ldots, m,
$$

and some constant $c_{j}>0$. This completes the proof of the theorem.

We can easily generalize Theorem 2 to the following system of nonlinear parabolic equations

$$
\left\{\begin{array}{c}
\partial_{t} u_{j}-L_{j} u_{j}=f_{j}(t, x, u) \\
\left.B_{j} u_{j}\right|_{\partial \Omega}=0
\end{array}\right.
$$

where $L_{j}$ and $B_{j}$ are elliptic operators and boundary operators respectively satisfying

$$
\begin{aligned}
& L_{j}=\sum_{\alpha \leq 2} A_{j, \alpha}(x) D^{\alpha}, \\
& B_{j} u=b_{j, 1} \frac{\partial u}{\partial n}+b_{j, 0} u .
\end{aligned}
$$

We denote by $D^{\alpha}=\partial_{x_{1}}^{\alpha_{1}} \ldots \partial_{x}^{\alpha}{ }_{n}^{n}, \alpha=\left(\alpha_{1}, \ldots, \alpha_{n}\right)$ and $|\alpha|=\alpha_{1}+\ldots{ }^{s}+\alpha_{n}$.

Furthermore, we assume that the principal parts of $L_{j}$ be

$$
P_{j}=\sum_{|\alpha|=2} A_{j, \alpha}(x) D^{\alpha}
$$

such that $A_{j, \alpha} \in C^{|\alpha|}(\bar{\Omega})$ and $b_{j, i} \in C^{1}(\bar{\Omega})$ are real, and $L_{j}$ are self-adjoint operators such that $\operatorname{ker}\left(L_{j}-c_{j}\right) \stackrel{j, \alpha}{=}\{0\}$ for some real $c_{j}$. Denote by $\sigma\left(L_{j}\right)$ the spectrum of $L_{j}$, for $j=1, \ldots, m$ and replace the assumption $(C I I)$ by the following,

$(C I I)^{\prime}$ The matrix $\mathbf{D}(f)=\left(f_{i, j}\right)$ is diagonalizable with eigenvalue $\mu_{j}$ and for every $j=1,2, \ldots, m$, there exists some integer $i(j)$ such that $\lambda_{j, i(j)-1}<\mu_{j}<\lambda_{j, i(j)}$, where $\left\{\lambda_{j, k}\right\}_{k=1}^{\infty}$ are the eigenvalues of the operators $L_{j}$.

Then by the same argument as used for Theorem 2, we have the following results.

Theorem 3: If $u$ is a $\mathbb{C}^{2}(\bar{\Omega}), \mathbb{L}^{2}$-bounded solution of equation (11), and if $f$ is a continuous function satisfies conditions $(C I),(C I I)^{\prime}$, then $u$ is $\mathbb{L}^{2}$-almost periodic.

Consider the solution $u$ of equation (11) in the sense that $u \in C^{1}\left(\mathbb{R}, \mathfrak{H}^{2}\right)$, where $\mathfrak{H}^{2}=H_{1}^{2}(\Omega)$ $\times, \ldots, H_{m}^{2}(\Omega)$, and

$$
H_{j}^{2}=\left\{u \mid u \in W^{2,2}(\Omega) \text { is real, and }\left.B_{j} u\right|_{\partial \Omega}=0\right\}
$$


Here $W^{2,2}(\Omega)$ is the Sobolev space (cf. Ladyženskaja [3]). Let the operator $\mathcal{L}=\left(L_{1}, \ldots, L_{m}\right)$, $\ell: \mathbb{L}^{2} \rightarrow \mathbb{L}^{2}$ with domain $D(\mathcal{L})=\mathfrak{J}^{2}$. Then we have a similar result as Theorem 2 (cf. Yang [4]).

Theorem 4: If $u$ is a $C^{1}\left(\mathbb{R}, \mathfrak{H}^{2}\right)$, and $\mathbb{L}^{2}$-bounded solution of equation, and if $f$ satisfies conditions $(C I),(C I I)^{\prime}$, then $u$ is $\mathbb{L}^{2}$-almost periodic.

Remark: The condition (CII) implies the uniqueness of $\mathbb{L}^{2}$-bounded solution to problem (1).

To prove the uniqueness, we assume that $u, v$ are the solution to problem (1), and let $w_{j}=u_{j}-v_{j}$ then $w=\left(w_{1}, \ldots, w_{m}\right)$ satisfies

$$
\partial_{t} w_{j}-\Delta w_{j}=\sum_{i} f_{j, i}\left(t, x, \Psi_{j, i}\right) w_{i}
$$

Applying Lemma 1, we see that

$$
M \cdot w \leq 0
$$

where $M$ is defined as in equation (10). By linear algebra, we obtain $w \equiv 0$.

\section{References}

[1] Corduneanu, C., Almost periodic solution to nonlinear elliptic and parabolic equation, Nonlinear Anal.-TMA (1983), 357-363.

[2] Foias, C., and Zaidman, S., Almost periodic solutions of parabolic systems, Ann. Scuola Norm. Sup. Pisa (1961), 247-262.

[3] Ladyženskaja, O.A., Solornikov, V.A., and Uralćeva, N.N., Linear and Quasilinear Equations of Parabolic Type, Amer. Math. Soc. Trans. of Math. Monographs, 1968.

[4] Yang, Y., Almost periodic solutions of nonlinear parabolic equations, Bull. Aust. Math. Soc. (1988), 231-238. 


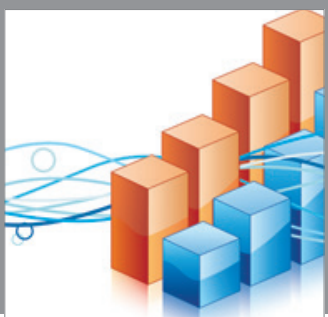

Advances in

Operations Research

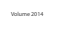

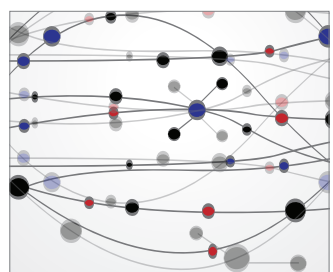

\section{The Scientific} World Journal
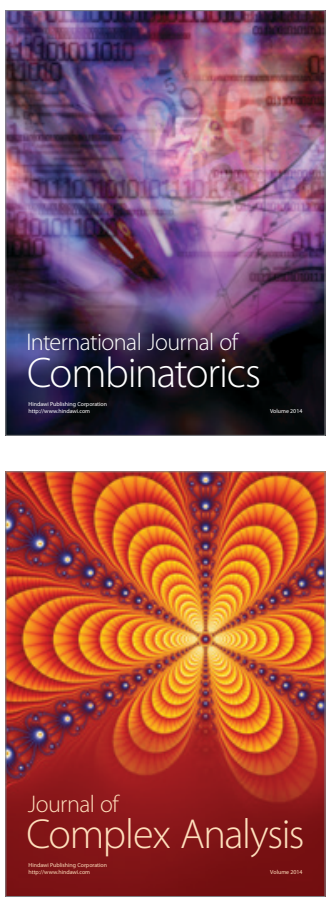

International Journal of

Mathematics and

Mathematical

Sciences
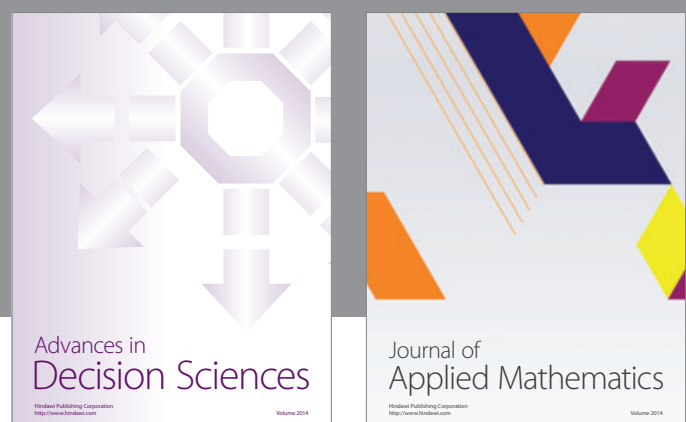

Journal of

Applied Mathematics
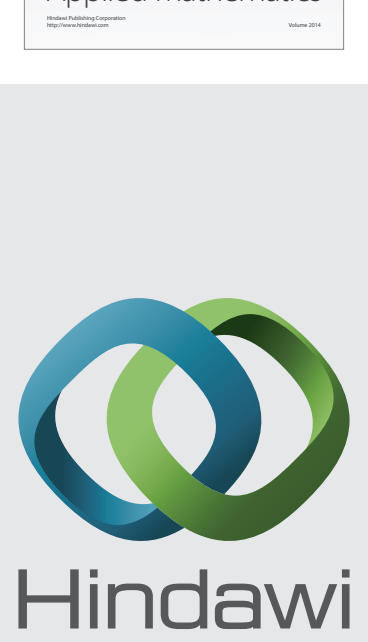

Submit your manuscripts at http://www.hindawi.com
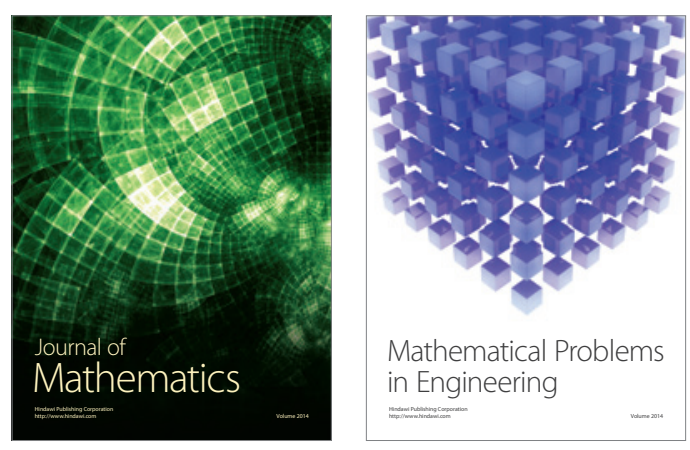

Mathematical Problems in Engineering
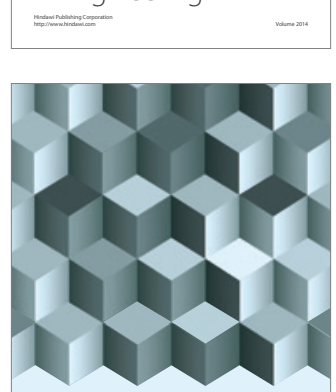

Journal of

Function Spaces
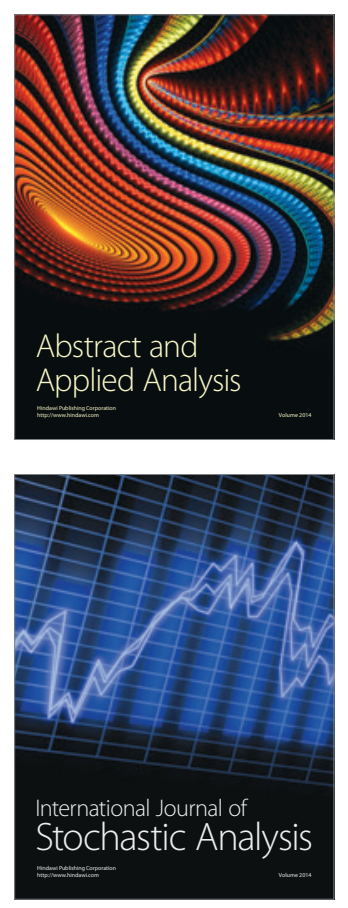

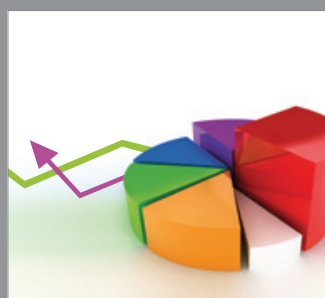

ournal of

Probability and Statistics

Promensencen
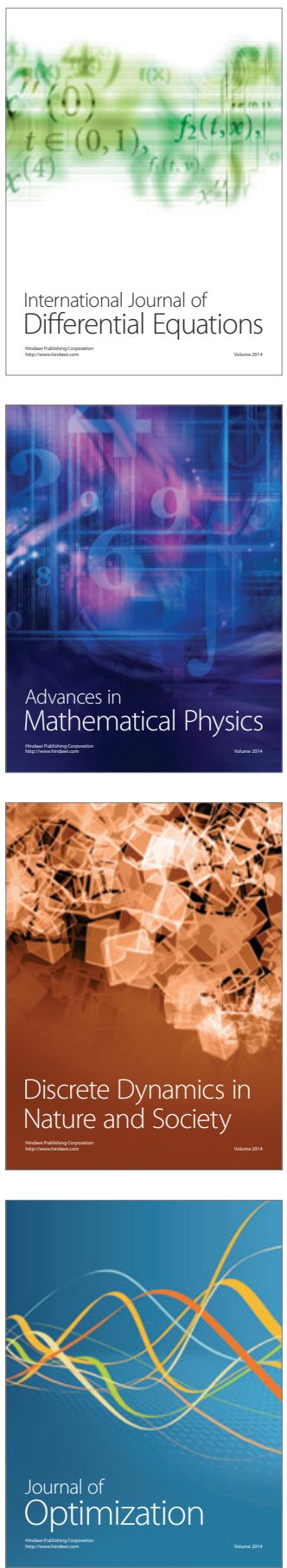